\title{
Chapter 19 \\ Establishing a Regional Digital Health \\ Interoperability Lab in the Asia-Pacific \\ Region: Experiences \\ and Recommendations
}

Philip Christian C. Zuniga, Susann Roth, and Alvin B. Marcelo

\begin{abstract}
Digital health is quickly evolving and bears great promises to strengthen health systems and quality of care. This chapter describes the establishment of a regional reference digital health interoperability laboratory in the Asia-Pacific region. It will explain the rationale for and process of establishing the laboratory. The chapter will also present the various services offered by the Standards and Interoperability Lab-Asia, key achievements to date and recommendations that can be used by other countries and regions for setting up their own interoperability labs.
\end{abstract}

Keywords Interoperability $\cdot$ Digital health $\cdot$ Standards $\cdot$ Electronic health records

\section{Learning Objectives}

By the end of this chapter, you will be able to:

- Describe the gaps and the challenges of current national healthcare systems in implementing digital health platforms and technologies.

- Enumerate the components of the GAPS interoperability framework.

- Analyze the OpenHIE Architecture.

- Evaluate the importance of co-creation in the development of national programs.

\subsection{Introduction}

The Asia eHealth Information Network (AeHIN) was established in 2011 to serve as a platform for countries in the Asia-Pacific region to share experiences, knowledge, and skills in the field of digital health. The World Health Organization (WHO) has

P. C. C. Zuniga ( $₫)$

SIL-Asia, Mandaluyong, Philippines

e-mail:phil@sil-asia.org

S. Roth

Asian Development Bank, Mandaluyong, Philippines

A. B. Marcelo

Asia eHealth Information Network, Manila, Philippines

(C) The Author(s) 2020

L. A. Celi et al. (eds.), Leveraging Data Science for Global Health,

https://doi.org/10.1007/978-3-030-47994-7_19 
promoted in-country digital health implementations as these implementations have proven to provide better health services on the ground level, and better decision making in the top government levels (WHO 2012). Since most members of AeHIN are country representatives from Ministries of Health $(\mathrm{MOH})$, much of the focus is on the development of national health information systems (HIS), clinical registries, electronic health records (EHRs), Citizen Registration and Vital Statistics (CRVS), and national dashboards for health sector planning (Fig. 19.1).

Digital health is relatively novel, so there has been constant evolution to leverage new technologies to new clinical scenarios. Unfortunately, with the rapid innovations, there has been an absence of widely adopted standards. Given that, within in any national system there will undoubtedly be many different technologies and platforms in use, resulting in restricted data silos, causing:

(1) Duplication in patient data entry

(2) Un-synchronized and inconsistent data

(3) Un-coordinated patient care.

And these issues are not only unique to few countries, these have been the pain points that countries have been sharing during regular AeHIN meetings. It is through this meetings, that interoperability became a key word for digital health implementors in the region. How will they attain interoperability in digital health?

To attain interoperability, AeHIN has developed the Mind the GAPS-Fill the GAPS framework. GAPS stand for Governance, Architecture, Program Management and Standards and Interoperability. The general idea to this framework came from AeHIN membership countries and development partners' experience in implementing their own ICT systems. Several AeHIN consultation meetings have been organized let by the AeHIN Governing Council which shaped the GAPS framework. As a practical solution to support digital health in countries, AeHIN has formally

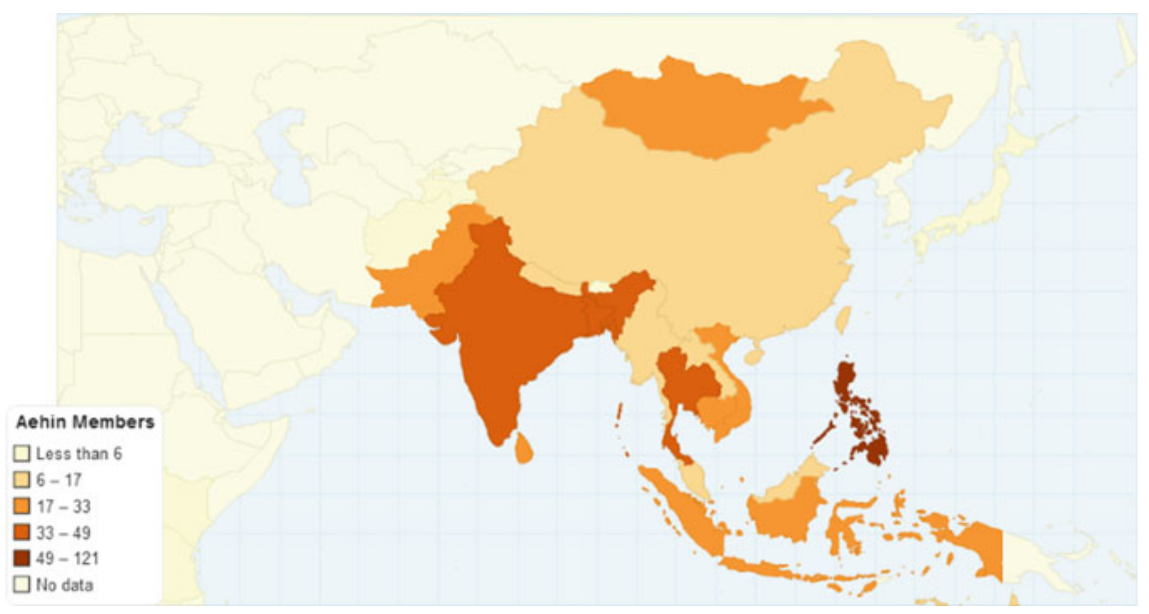

Fig. 19.1 AeHIN Country memberships 
launched the development of the Community of Interoperability Labs (COIL) at the 4th AeHIN meeting in Bali. COIL is being developed to serve the needs of the region in implementing interoperable solutions based on globally identified architectures (Marcelo 2015). COIL will also provide support for the technical implementation of GAPS to whom and what are the services.

COIL will be composed of country level interoperability laboratories/centers of excellence. It is envisioned that the centers will implement digital health prototypes and test systems to make it easier for the MOHs to implement digital health solutions. In October 2016, AeHIN signed a Memorandum of Agreement with UP Manila to house the AeHIN interoperability laboratory (Hexagon 2016). The Asian Development Bank (ADB) supports the newly formed interoperability lab financially and with strategic guidance. The laboratory was eventually named as the Standards and Interoperability Lab-Asia (SIL-Asia). SIL-Asia was initially housed at the Expanded Health Research Office, at the Philippine General Hospital.

\subsection{Project Narrative}

Countries are investing in Digital Health as a core component to achieve universal healthcare coverage (UHC). Digital Health is seen an enabler to UHC because having ICT systems in place would potentially enable health care providers to streamline their work and focus on more important matters. At the same time, ADB has been investing heavily and leading knowledge networks in digital health governance, unique health identification, and health standards and interoperability. Because of this support, it was envisioned as a space where technology can be tested without bias and innovations can be widely developed from meaningful research. Since ADB already has made investments in digital health through planning, procurement, development, and implementation of software systems, they logically concluded it be important that they support the development of an interoperability lab to mitigate risks. An example of how a lab can support the bank in risk mitigation is by providing technical expertise in the development of tenders and terms of reference for software procurements hence preventing possible problems that will come along the way.

A- Process and Scope

The development of the lab required the establishment of different components that are the following: (ADB CMS 2018)

(1) Build an Interoperability laboratory.

(2) Build adapters, libraries, mediators and other assets to facilitate integration of various systems including EMR, PMS, HMIS, and databases that support various health programs.

(3) Support AeHIN countries in making their applications compliant with the AeHIN reference architecture.

(4) Conduct Interoperability testing procedures. 
(5) Provide feedback to other labs (Mohawk) regarding issues discovered in the implementation of the laboratory.

(6) Provide feedback to laboratory manager to help in refining the standard operating procedures and management structure of the interoperability laboratory.

(7) Document all source codes produced by the laboratory.

B- Human Resources

Laboratory manpower have changed from time to time, but throughout the past two years, SIL-Asia has maintained a core of around 7-8 experts (Table 19.1).

Among the capacity development program that the members of SIL-Asia has taken are as follows:

(1) Interoperability Lab Training-The members of the Laboratory were trained on how to build up the laboratory and on how digital health standards such as HL7 can be used as leverage for digital health development.

(2) IHE Training - The members of the Laboratory trained on IHE profiles, profile development process, testing and organization.

(3) Standards Training - The members of the Laboratory trained on HL7-FHIR standard. It includes training on setting up a server, creating applications and working FHIR APIs.

C- Partnerships

International Experts and partners were also brought into ensure that laboratory members understand the technology trends in digital health all around the world. The goal of bringing in experts and partners is to ensure that the capacity of the members of SIL-Asia will be developed and will make the laboratory navigate the digital health perspectives from all over the world. Collaborations include partnerships with

Table 19.1 Composition of SIL-Asia

\begin{tabular}{l|l}
\hline Position & Roles \\
\hline Laboratory director & Handles the overall direction of the lab \\
\hline Technical lead & $\begin{array}{l}\text { Manages the day to day activities of the lab. Makes the decisions } \\
\text { on the technical activities of the lab }\end{array}$ \\
\hline Health lead & $\begin{array}{l}\text { The lead clinician member of the lab. Reviews all clinical related } \\
\text { activities in the lab }\end{array}$ \\
\hline Laboratory experts & \multicolumn{2}{|l}{ Governance, architectures, digital health investment } \\
\hline Terminologies expert & Terminology services \\
\hline Standards expert & FHIR, HL7 CDA \\
\hline Health systems experts & Dashboards, EMRs, EHRs \\
\hline Emerging technologies expert & AI, Blockchain, data science \\
\hline Web technologies expert & Web infrastructure, APIs, REST, SOAP \\
\hline Interoperability expert & ESBs, mediators, centralized interoperability layers \\
\hline
\end{tabular}


the IHE (Integrating Healthcare Enterprise community), HL7-FHIR community, World Health Organization.

\subsection{Laboratory Goals and Services}

SIL-Asia services revolve around the laboratory's underlying objective of having [1] interoperable digital health applications, [2] innovative digital health solutions and [3] impactful digital health investments. These objectives can be achieved with the Four T's framework. This framework has also been used by other interoperability labs in their own service catalogs [Medic Lab, Jembi Lab] (Fig. 19.2).

Testing: SIL-Asia has the vision of becoming the primary standards testing and health software benchmarking center in the region for the public sector. As testers, the laboratory can check if software uses existing international standards via a testing protocol that is developed internally. The laboratory also performs benchmarking of existing technologies, via a quantitative scoring framework. Will it be possible to share more details about the scoring? This would be very helpful.

Tooling: The laboratory develops and maintains several tools that it shares with various partners in the region. The tools developed by the laboratory include publications, software tools, and cloud deployed software. Are the tools open source? How are they developed? What are the core functionalities?

Teaming: SIL-Asia members can also participate in actual software development projects or in actual implementation of solutions. Most of the times, this is done to reduce the risk from the actual developers since SIL-A can take some of the risks away by doing testing and quality control during an earlier part of the project.

Training: SIL-Asia organizes trainings for various digital health partners. The training either includes SIL-Asia members doing the trainings or international experts

Fig. 19.2 SIL-Asia services framework

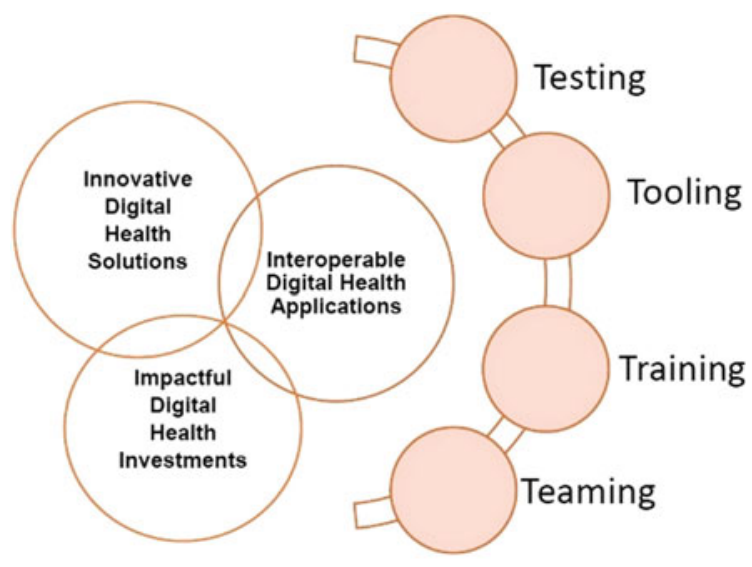


Table 19.2 SIL-Asia services and outputs

\begin{tabular}{l|l|l}
\hline Activity & Classification & Date \\
\hline Support to Navotas City computerization & Teaming & March 2017-December 2017 \\
\hline Support to PGH computerization & Teaming & March 2017-present \\
\hline $\begin{array}{l}\text { Testing and certification of iClinicSys as } \\
\text { compliant to RxBox standards }\end{array}$ & Testing & April 2017-October 2017 \\
\hline Governance deep dive in Navotas & Training & June 2017 \\
\hline Interoperability deep dive series & Training & August 2017-October 2017 \\
\hline Costing tool implementation & Tooling & March 2017-December 2017 \\
\hline Digital health impact framework & Tooling & February 2018-December 2018 \\
\hline SIL-Asia lab on cloud & Tooling & November 2017-present \\
\hline $\begin{array}{l}\text { Support to FHIR implementation in Viet } \\
\text { Nam }\end{array}$ & Training & October 2017-present \\
\hline DHIF training & Training & February 2018-present \\
\hline $\begin{array}{l}\text { Development of health enterprise } \\
\text { architecture framework }\end{array}$ & Tooling & August 2018-present \\
\hline FHIR server & Tooling & June 2018-present \\
\hline
\end{tabular}

are hired to conduct the training in partnership with the SIL-A team. This pairing approach allows also for fast capacity development of the SIL-A team (Table 19.2).

\subsection{Technical Aspects of the Laboratory}

As a Digital Health Interoperability Lab, it is important for the laboratory technical capabilities on various digital health applications and standards. Currently, the laboratory is focused on three specific areas:

(1) Health Information Exchange (HIE) implementations - this involves the installation, deployment and benchmarking of key HIE components. The following are the reference components that are installed in the SIL-A cloud.

Medic CR (GitHub 2018a) for Client Registry

OpenEMPI (Open-mpi.org 2018) for Client Registry

OpenInfoMan (GitHub 2018b)for Facility and Health Worker registry

WSO2 ESB (Wso2.com 2018) for Interoperability Layer

OpenHIM (Openhim.org 2018) for Interoperability Layer

DHIS2 (GitHub 2018c) for HMIS

OpenMRS (Openmrs.org 2018) for Health Information System

HL7 CDA (H17.org 2018) and HL7 FHIR (HL7 FHIR 2018) for data exchange standards

These software are integrated together using the different kind of HIE frameworks. (i.e. OpenHIE) (Fig. 19.3) 


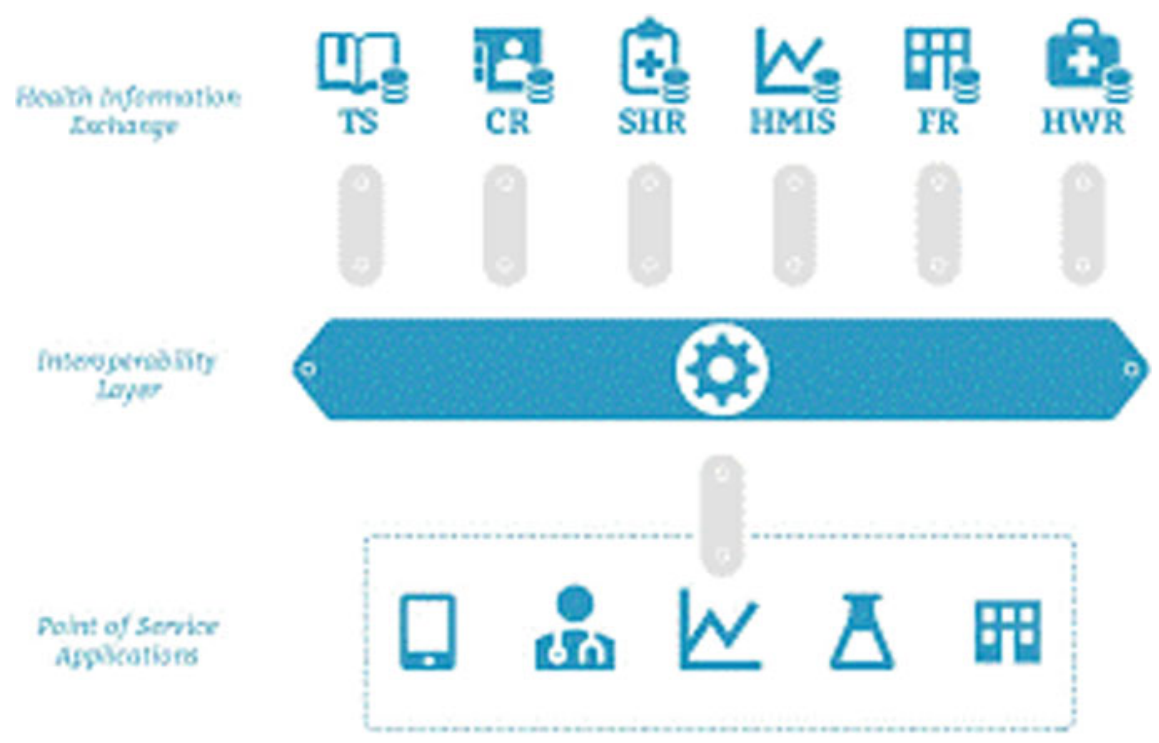

Fig. 19.3 OpenHIE architecture

(2) FHIR Server Implementation-The Laboratory has also invested on setting up a FHIR server that can be used in the region. This is a timely resource as many countries in Asia are looking at implementing FHIR. FHIR is the emerging standard right now in medical and health data exchange. The server that the lab has can be used by countries in order for them to see how FHIR works.

(3) Emerging Technologies - The Laboratory is looking at emerging technologies as possible solutions to the digital health issues of LMICs (Fig. 19.4).

As part of SIL-Asia's tooling services, the laboratory developed a demonstration material that demonstrate the integration of several health information systems using IHE profiles, HL7 CDA and HL7 FHIR standards. These integration efforts were also used in populating data in the dashboard. The demonstration showed how Interoperability works, how foundational investments are important, and the need of having in-country interoperability laboratories.

\subsection{Non-technical Interoperability}

Interoperability solutions are not only technical, but there are also non-technical aspects as well. The Laboratory has worked on knowledge products on health IT governance, digital health investment analysis, costing tools and health enterprise architecture. Technical solutions should always conform with non-technical solutions as interoperability frameworks are derived from non technical components. 


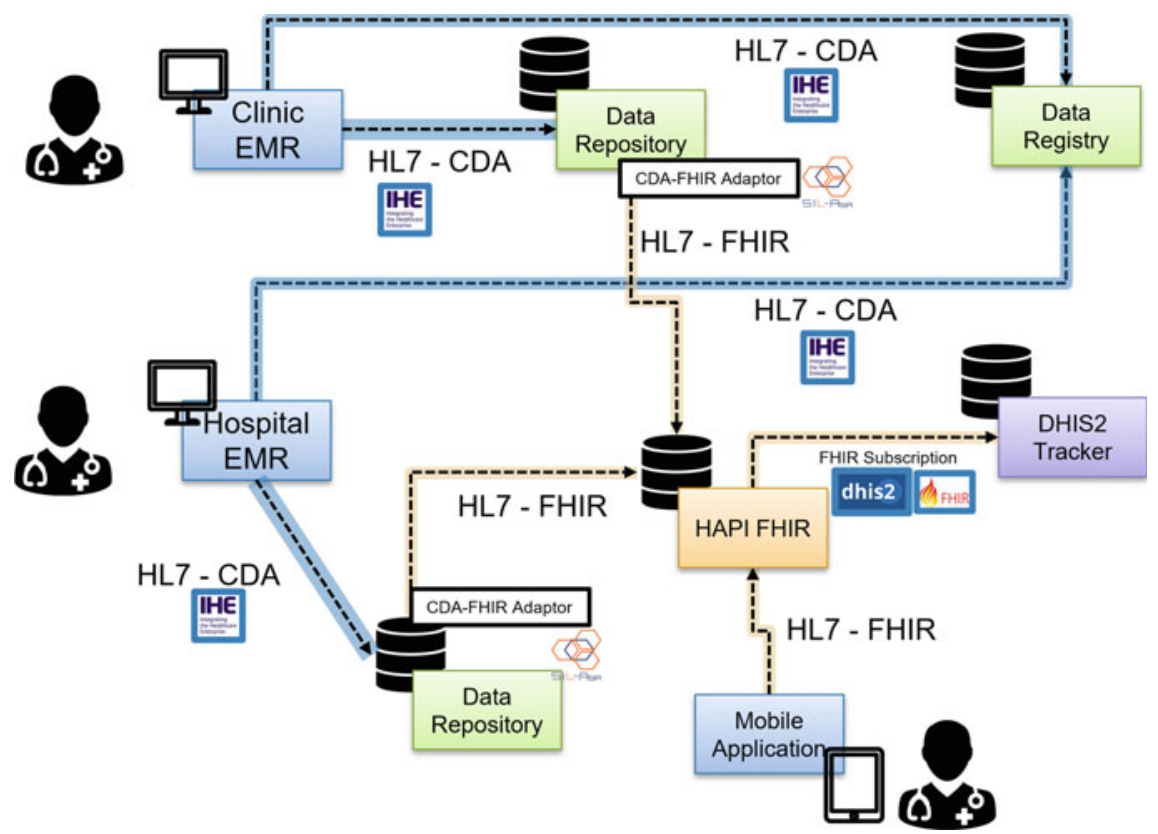

Fig. 19.4 Blueprint of SIL-Asia integration demo for AeHIN 2018

Governance drives investments in digital health. Investments in digital health should be in accordance to a governance mandated health enterprise architecture. The choice of technical solutions will then be based on the architecture and on the affordability of a solution.

In this framework SIL-Asia has taken a whole of the picture approach to digital health solutions. Since most of the services are expected to be subscribed by MOHs, it is important that there is focus at Governance and Architecture before the actual solutions. The same approach is parallel to AeHIN's Mind the GAPS_Fill the GAPS approach.

\subsection{Co-creation Framework}

One of the important functions of the laboratory is to help in setting up labs in other countries. SIL-Asia follows AeHIN's philosophy of "If you help friends, friends will help you." In setting up Laboratories, the laboratory values the fact that countries should claim ownership and responsibility of their own laboratories. Hence Laboratories are not established by the SIL-Asia alone, but we co-create with other countries (Fig. 19.5).

The teaming framework, which underpins the COIL, relies on the co-creation process and is consisting of three components. 
- Regular communication and knowledge sharing

- Sharing of artifacts and best practices.
- Conduct a situational analysis of country needs vis-à-vis Health SDGs.

- Articulate Vision Mission

- Set Key Performance Indicators - Build Governance Structure

\section{Co-Learning}

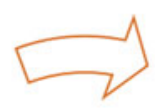

Corealialization

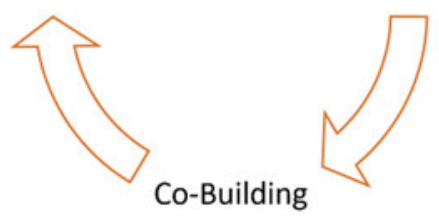

- Capacity Building on Lab Governance

- Capacity building on Lab Investments

- Capacity building on technologies and architectures

Fig. 19.5 Co-creation framework

1. Co-realization: In co-realization the laboratory identifies the drivers, mission and vision of countries for developing their own laboratories. The next step is to develop the key performance indicators and finally, before actual implementation, a governance structure for decision making and for the laboratory strategic directions needs to be established.

2. Co-building: In co-building, SIL-Asia assists the laboratory in building their structures. This include building up their own investments and technologies/architecture. SIL-Asia share its manual of laboratory operations to ensure that the new in-country laboratory can build on from SIL-Asia's experiences.

3. Co-learning: Co-learning is the heart of SIL-Asia's co-creation framework. In co-learning, COIL members are expected to share experience, knowledge, technologies and expertise with one another. In sharing these, SIL-Asia as part of COIL enables a fast set up and capacity development of new in-country labs.

\subsection{Achievements, Results and Conclusions}

During the past two years, SIL-Asia, with support from the ADB, has produced five (5) tools that have contributed to the general AeHIN community. The tools have been made available and can be accessed via the SIL-Asia website:

- Digital Health Impact Framework-Presented to 6 countries. Discussed as a side meeting during the 2018 AeHIN GM where around 42 countries have their representatives. Publication of the DHIF by the ADB is expected by end of 2018 . 
- Costing Tool—An accompanying tool to DHIF. Presented to 6 countries.

- Health Enterprise Architecture Framework-To be published by 2018. The paper is an output from a workshop attended by four AeHIN countries.

- Lab in the Cloud-A cloud based set up that demonstrate various digital health software and how they are interoperable with another. The cloud set up includes a version of OpenMRS based EMR, a WSO2 ESB instance, a Medic Client Registry instance, a DHIS2 instance and a HAPI FHIR server instance.

- AeHIN FHIR Server-SIL-Asia also have set up a FHIR server that can be used by the AeHIN region. It was observed that majority of the usage of the server is from Philippines, Viet Nam and Taipei, China.

SIL-Asia has also organized various trainings that were attended by a number of participants. The following are the key trainings organized by SIL-Asia.

\begin{tabular}{l|l|l}
\hline Interoperability deep dives & August 2017-September 2017 & 20 people (on site) \\
\hline $\begin{array}{l}\text { Webinars on } \\
\text { Interoperability }\end{array}$ & December 2017-January 2018 & $\begin{array}{l}\text { Approx. 30 people average } \\
\text { (online) }\end{array}$ \\
\hline $\begin{array}{l}\text { HL7 FHIR Training, Viet } \\
\text { Nam }\end{array}$ & March 2018 & 50 people (onsite) \\
\hline $\begin{array}{l}\text { Advanced FHIR Training, } \\
\text { Viet Nam }\end{array}$ & June 2018 & 50 people (onsite) \\
\hline $\begin{array}{l}\text { HL7 FHIR Training for } \\
\text { EMRs, Philippines }\end{array}$ & August 2018 & 20 people (onsite) \\
\hline
\end{tabular}

from the trainings, SIL-Asia has been involved in presenting several of SILAsia's knowledge products on Governance, Architecture, Blockchain in international symposiums (AeHIN Knowledge exchange Thailand, AeHIN-Taiwan Knowledge Exchange, Taiwan, Big Data Conference, Philippines, AeHIN 2018, Colombo).

\subsection{Community of Interoperability Labs}

One significant output of SIL-Asia is the establishment of COIL. It has always been the vision of the laboratory to help countries establish their own in-country labs. The original reason why the laboratory was established was because AeHIN needed a pilot laboratory that can be used as blueprint by countries to understand the steps and the value of establishing their own laboratories. By the end of 2018, it is expected that around eleven (11) countries will establish/planned for their own laboratories:

(1) Philippines-Since SIL-Asia is based in Manila, it has become the de facto interoperability laboratory for the Philippines. It has supported various Philippine based health stakeholders such as the Philippine General Hospital, the Department of Health, the Navotas LGU, the National Telehealth Center, the eHatid EMR. Plans are on the way for SIL-Asia to lead in proposing for a local Philippine Interoperability Lab. 
(2) Viet Nam-The eHA Viet Nam has requested for support for the establishment of an interoperability lab that focuses on testing software systems. In response SIL-Asia has been actively supporting Viet Nam. Several capacity development measures have been supported.

(3) The following countries have indicated interest in setting up their own respective laboratories. It is expected that full blown capacity development activities with these laboratories will commence by early 2019 .

a. Malaysia

b. Thailand

c. Indonesia (with the role out of the national health insurance and investments in health service supply, better health information system management is required which provides real time data for planning and decision making, which improves the continuum of care and which helps to improve quality of care)

d. Bangladesh

e. Nepal

f. Taipei, China

g. Myanmar

h. Mongolia

i. India.

During the conclusion of the 2018 AeHIN GM, a commitment ceremony was held by COIL members. The commitment focuses on knowledge, technology and expertise sharing (Fig. 19.6).

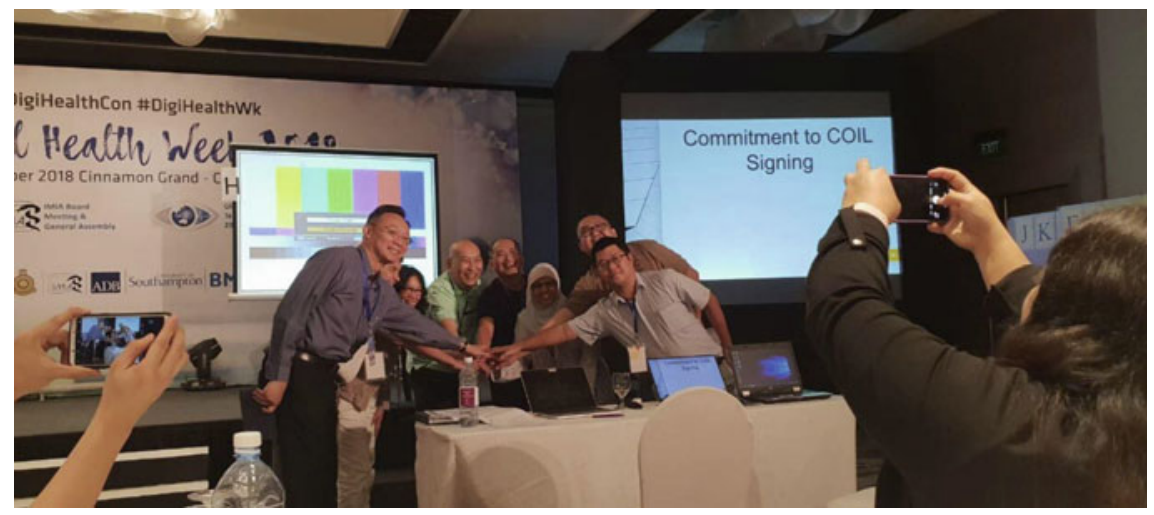

Fig. 19.6 COIL commitment ceremony 


\subsection{Recommendations}

Learning from the SIL-A experience, the following recommendations have been developed for countries implementing an interoperability laboratory.

(1) Interoperability Laboratories should be part of the Country's digital health infrastructure. This is important so that their recommendations can be taken into consideration when countries decide on their digital health solutions and investments.

(2) Interoperability laboratories must have full time, dedicated staff and each staff needs a focus area with a defined capacity development plan.

(3) Laboratories should have a good administrative and communications support. This enables the laboratory experts to focus on the technical work while the communications and administration expert handles the knowledge management.

(4) Laboratories, if they are not inside existing budgeted infrastructures need to consider sustainability early-on. In the case of SIL-Asia, it is planned to set up a non-profit entity for the laboratory operations so that it can receive funding from multiple external sources, charge for services provides for private sector digital health providers, or apply for research grants from research entities.

(5) A challenge that the lab is facing is funding. Right now, the lab functions efficiently due to the support of the ADB, however, to be more sustainable, it should look to partner with different development partner.

(6) The lab should also expand to emerging technologies such as Artificial Intelligence and Data Science, as these are the next targets once interoperability is attained. If there is an interoperability with systems, it will be easier to collect data and hence once more data is collected analytics can be done and hence these analyses can lead to important results to AI or data science.

Acknowledgements SIL-Asia is powered by the Asia eHealth Information Network with support from the Asian Development Bank. The funds of the laboratory primarily came from the ADB People's Republic of China Poverty Reduction and Regional Cooperation Fund. Additional support was provided by the World Health Organization, the Norwegian Aid Agency and UNICEF.

\section{References}

ADB CMS. (2018). TOR for Health Interoperability Expert. Retrieved October 11, 2018, from https://uxdmz06.adb.org/OA_HTML/OA.jsp?page=/adb/oracle/apps/xxcrs/opportunities/ webui/OppPG\&OAPB=ADBPOS_CMS_ISP_BRAND\&_ti $=1442422620 \& O A M C=80315$ _ 40_0\&menu=Y\&oaMenuLevel=1\&oapc $=6 \&$ oas $=w P 7 n I j d K c 71 T F K E R c 45 n B A$.

GitHub. (2018a). MohawkMEDIC/client-registry. Retrieved October 11, 2018, from https://github. com/MohawkMEDIC/client-registry/wiki/Installing-the-MEDIC-CR-on-openSUSE-13.2.

GitHub. (2018b). Openhie/openinfoman. Retrieved October 11, 2018, from https://github.com/ope nhie/openinfoman.

GitHub. (2018c). DHIS 2. Retrieved October 11, 2018, from https://github.com/dhis2. 
Hexagon. (2016). COIL Visits UP Manila.

HL7 FHIR. (2018). HL7 FHIR. Retrieved October 11, 2018, from https://www.hl7.org/fhir/.

H17.org. (2018). HL7 Standards Product Brief-CDA® Release 2. Retrieved October 11, 2018, from http://www.h17.org/implement/standards/product_brief.cfm?product_id=7.

Marcelo, A. (2015). Proceedings of MA4HAP. Bali, Indonesia: AeHIN.

Openhim.org. (2018). OpenHIM-simplifying interoperability. Retrieved October 11, 2018, from http://openhim.org/.

Open-mpi.org. (2018). Open MPI: Open Source High Performance Computing. Retrieved October 11, 2018, from https://www.open-mpi.org/.

Openmrs.org. (2018). OpenMRS. Retrieved October 11, 2018, from https://openmrs.org/.

WHO. (2012) The WHO ITU National eHealth Strategy Toolkit.

Wso2.com. (2018). WSO2 Enterprise Service Bus-The Only 100\% Open Source ESB. Retrieved October 11, 2018, from https://wso2.com/products/enterprise-service-bus/.

Open Access This chapter is licensed under the terms of the Creative Commons Attribution 4.0 International License (http://creativecommons.org/licenses/by/4.0/), which permits use, sharing, adaptation, distribution and reproduction in any medium or format, as long as you give appropriate credit to the original author(s) and the source, provide a link to the Creative Commons license and indicate if changes were made.

The images or other third party material in this chapter are included in the chapter's Creative Commons license, unless indicated otherwise in a credit line to the material. If material is not included in the chapter's Creative Commons license and your intended use is not permitted by statutory regulation or exceeds the permitted use, you will need to obtain permission directly from the copyright holder. 